\title{
Acercamiento antropológico de la alimentación y salud en México
}

\section{$\mid{ }^{1}$ Miriam Bertran Vilà |}

Resumo: En este artículo presento un panorama de la alimentación en México, particularmente sobre la ciudad de México desde una perspectiva antropológica considerando aspectos socioculturales y económicos. Inicia con una breve revisión de los estudios antropológicos sobre alimentación en México, para reconocer tanto los aportes metodológicos como los principales problemas de estudio. Posteriormente, se presentan algunos datos de contextualización del país y de la ciudad que enmarcan los datos nutricios y alimentarios característicos. En las siguientes secciones se proponen algunas explicaciones sobre algunos de los fenómenos alimentarios contemporáneos, donde la obesidad es la característica principal en una sociedad de reciente acceso al consumo masivo, al mismo tiempo que se enfrenta al ideal cultural de delgadez. Los datos sobre la alimentación en México y los fenómenos sociales relacionados dan cuenta de la complejidad del fenómeno alimentario y de cómo los procesos macrosociales afectan las decisiones cotidianas de la gente. El análisis antropológico de la alimentación en la población mexicana ha permitido mostrar la relación entre estos procesos históricamente y en fechas recientes. Son la muestra de la utilidad de la metodología antropológica para estudiar la alimentación contemporánea, llena de contradicciones, que tienen que ver con el desarrollo del capitalismo y la sociedad de consumo, la promoción al consumo, y el acceso inmediato a él, la medicalización de la vida cotidiana, las ideas sobre el control corporal, y la imagen como un elemento de estatus.

> Palavras-chave: Alimentação; Cultura; México; Antropologia.
1 Profesora-investigadora. Departamento de Atención a la Salud. Universidad Autónoma Metropolitana-Xochimilco. México. Correo electrónico: mbertran@correo.xoc.uam.mx 


\section{Introducción}

México es un país grande y diverso tanto en términos económicos como sociales y culturales. Desde la segunda mitad del siglo pasado ha ido experimentando, cada vez con mayor rapidez, cambios en todos los aspectos de su vida. Conviven al mismo tiempo la globalización y todos sus efectos, con estilos de vida tradicionales de los grupos indígenas. Las diferencias socioeconómicas se advierten en los niveles de pobreza, según algunos autores hasta casi el 70\% (DAMIÁN; BOLTVINIK, 2003); al mismo tiempo hay población con niveles de riqueza altísimos, entre los que se cuenta uno de los hombres más ricos del mundo. En términos culturales, la diversidad se expresa, entre otras cosas, en la cantidad de lenguas indígenas que se hablan, en total 62, lo que convierte al país en el de mayor diversidad lingüística en América Latina. Así, la variedad de grupos de población es una combinación de factores socioeconómicos y étnicos. Estas diferencias, hacen que el acceso a los alimentos, tanto en términos económicos de producción, distribución y compra, como en términos de la accesibilidad cultural sea muy diversa.

En términos nutricionales, México es el segundo país del mundo con población con sobrepeso y obesidad. Según la última encuesta de salud y nutrición (ENSALNUT, 2006) más del 70\% de la población mexicana adulta tiene exceso de peso; como consecuencia de esta situación nutricia la diabetes mellitus II está entre las primeras causas de muerte. El crecimiento de la obesidad y sus consecuencias epidemiológicas en los últimos veinte años coincide con el crecimiento económico. Un sector cada vez mayor de la población se ha incorporado al mercado de trabajo, a la economía monetarizada, se ha agudizado la urbanización y ha aumentado la disponibilidad de alimentos. Los efectos de la globalización se han manifestado en términos alimentarios en una mayor distribución de alimentos, locales y de otros lugares, y una mayor circulación de ideas sobre los alimentos. Todos estos aspectos parecen tener un impacto en la forma de comer de la población, particularmente en las zonas urbanas donde los cambios han sido más agudos y más rápidos.

La complejidad del fenómeno alimentario actual y sus consecuencias nutricionales y epidemiológicas obligan a buscar opciones de análisis que permitan entender cabalmente el fenómeno. En este sentido, la antropología ofrece la posibilidad de estudiar la alimentación en su dimensión sociocultural, ya que como cualquier actividad humana está determinada por las características particulares de las 
poblaciones. Los estudios antropológicos sobre las formas de comer han aportado elementos para explicar por qué un grupo social come de determinada manera.

En este artículo presento un panorama de la alimentación en México, particularmente sobre la ciudad de México desde una perspectiva antropológica considerando aspectos socioculturales y económicos. Empezaré por una breve revisión de los estudios antropológicos sobre alimentación en México, para reconocer tanto los aportes metodológicos como los principales problemas de estudio. Posteriormente presento algunos datos de contextualización del país y de la ciudad que enmarcan los datos nutricios y alimentarios característicos. En las siguientes secciones propongo algunas explicaciones sobre algunos de los fenómenos alimentarios contemporáneos. Debo aclarar que me voy a centrar en la ciudad de México por una parte porque es un buen ejemplo de los cambios alimentarios recientes, y por otra porque es donde he desarrollado mis proyectos de investigación desde 1996. El análisis de la alimentación desde esta mirada da la pauta para reflexionar sobre el papel de los fenómenos macrosociales en la situación nutricional actual, donde la epidemia de obesidad es la característica principal.

\section{Alimentación y antropología}

La alimentación es un fenómeno complejo en tanto que si bien cubre una necesidad biológica, no todos los grupos humanos la llevan a cabo del mismo modo. Los estudiosos sobre el tema coinciden en señalar que el "hecho alimentario" es tan complejo como la sociedad misma, y está determinado por una diversidad de factores sociales, culturales y económicos. Pero además, estos factores se ponen en juego de manera diferente según circunstancias específicas. Al respecto, Contreras y Gracia (2005) señalan que la alimentación no es una cuestión de hábitos, sino de comportamiento; es decir, no tiene que ver con la repetición de actos de una misma naturaleza, más bien con el conjunto de usos y cualidades que caracterizan el sistema alimentario de determinado grupo social.

En este sentido, Sydney Mintz (1996) plantea que los grupos humanos le dan a los alimentos atributos o significados para clasificarlos de manera que guían su elección según ocasión, condición socioeconómica, edad, sexo, estado fisiológico, imagen corporal, prestigio, entre otros. Mintz señala, que los significados pueden ser externos e internos. Los primeros se refieren a todas aquellas condiciones externas al ámbito doméstico que pueden intervenir sobre las decisiones domésticas como 
la disponibilidad y accesibilidad de los alimentos, horarios de trabajo, organización del tiempo, cuidado de los hijos, exposición a alimentos nuevos, por mencionar algunos. En esta categoría entrarían, por ejemplo, las ideas sobre alimentación y salud que emiten diferentes medios. Por su parte, los significados internos constituyen lo que quieren decir las cosas para quien las usa, volviendo familiar e íntimo las condiciones del contexto. Es decir, son la expresión de la adaptación doméstica a las condiciones externas y se expresan en las decisiones de ¿qué se come? ¿Dónde? ¿Cuándo? ¿Cómo se prepara? ¿Con quién? ¿Con qué? ¿Por qué? Además de todos estos elementos, hay que considerar que los alimentos proporcionan gusto y placer, sensaciones individuales pero también moduladas de manera grupal.

Como fenómeno cultural complejo, la antropología se ha acercado al estudio de la alimentación desde principios del siglo XX, a partir de varias perspectivas teóricas, a veces como un punto central de los estudios antropológicos, a veces como parte del análisis de otros fenómenos. En el caso de México, los inicios de los estudios antropológicos sobre la alimentación nos remiten a a Fray Bernardino de Sahagún, quien en la Historia General de las Cosas de la Nueva España (1985) hace una descripción detallada de las costumbres y la religión de los indios antes de la llegada de los españoles, incluyendo a las formas de producción y consumo de los alimentos, a partir del uso de la observación directa. También es importante destacar los relatos de los viajeros del siglo XIX, en los que muestran a detalle la vida cotidiana y como parte de ella, las formas de preparación y consumo de alimentos en México. Los datos de uno y otros, si bien no son aportaciones de la antropología profesional, constituyen una excelente fuente de información para el estudio de la alimentación en México.

Durante el periodo denominado "el porfiriato" (1877-1911) Francisco Bulnes, un periodista liberal de la época, desarrolló la teoría dietética de la raza, que sostenía que la inferioridad y superioridad raciales se daba según el alimentos básico preponderante, ya fuera trigo, arroz o maíz. A partir de esto explicó que la superioridad alcanzada por los pueblos europeos y el estadounidense se debía a que comían fundamentalmente trigo, y el atraso de los pueblos indios de México es resultado del alto consumo de maíz (ESPAÑA, 1988). La idea de Bulnes suena sumamente descabellada, pero bien a bien no se desmintió hasta que en los años cuarenta del siglo XX se realizaron estudios para conocer los aportes nutricionales de la dieta mesoamericana. 
El primer antropólogo mexicano con formación universitaria formal que tuvo interés en el asunto fue Manuel Gamio, quien dedicó su trabajo académico fundamentalmente a la búsqueda del mejoramiento de las condiciones de los indígenas. En el caso de la alimentación Gamio consideraba que los indígenas no tenían cubiertas sus necesidades biológicas, pues se alimentaba de maíz, chile y en menor proporción frijol. Esta situación de desnutrición provocaba altas tasas de mortalidad, poco desarrollo demográfico y bajo rendimiento en el trabajo físico, lo que se consideraba un problema para el desarrollo del México posrevolucionario. La poca variedad de la dieta, que carecía de lo que Gamio consideraba elementos indispensables como los que se hallan en la leche, la carne, el pan y los huevos, es atribuida a la pobreza (GAMIO, 1987). El interés de Gamio en el tema alimentario se manifiesta en la cantidad de artículos sobre el tema que apareció en América Indígena. Estos artículos, generalmente sobre comunidades indígenas campesinas, dan cuenta por una parte de la situación de la población y por otra de los intereses del Instituto sobre el tema; en este sentido, ambas cosas han ido cambiando. La alimentación indígena ha pasado de una dieta aparentemente monótona basada en la agricultura de subsistencia a una alimentación variada, determinada por los recursos económicos, los sistemas de comunicación y una más alta disponibilidad de alimentos industriales. En lo que se refiere a las preocupaciones del Instituto, en los años cuarenta y cincuenta, la alimentación aparecía como un paradigma del desarrollo, mientras que en los sesenta se planteaba que la dieta indígena está determinada por la estructura económica. En esta época también aparecen las primeras ideas sobre el valor simbólico de los alimentos que será más ampliamente explorada en los setentas y ochentas. La aculturación fue una preocupación ya que se consideraba que la introducción de nuevos alimentos generaría cambios indeseables en la dieta indígena provocando mayores problemas de nutrición. (LÓPEZ; VERA, 1988). Este tema fue también de interés en las reflexiones que sobre la alimentación hace Aguirre Beltran en su texto sobre antropología médica (AGUIRRE-BELTRAN, 1986).

En los años sesenta, el antropólogo mexicano Guillermo Bonfil se interesó por el tema y desarrolló su tesis de licenciatura sobre el hambre en Yucatán. Planteó un trabajo de investigación en antropología aplicada, que formaba parte de una investigación más amplia que el Instituto Nacional de la Nutrición estaba llevando a cabo en la zona. Sus objetivos fueron proporcionar elementos de orden social que 
permitieran un mejor entendimiento de los problemas del hambre en la zona de Sudzal en Yucatán y sirvieran como base para evaluar el programa del INN (Instituto Nacional de la Nutrición) para mejorar el estado de nutrición (BONFIL, 1962).

La preocupación por la alimentación en los años ochenta llevó a la realización de un seminario interdisciplinario en un proyecto de la UNAM "La alimentación del futuro" trató el tema desde diferentes disciplinas, incluyendo la antropología. La representación antropológica estuvo a cargo de Larissa Adler y Claudio Lomnitz (1988) quienes mostraban la importancia cultural de la comida con el fin de que los responsables de las políticas y programas de alimentación lo consideraran en sus decisiones. En los últimos veinte años, encontramos algunas tesis de grado en antropología acerca de la alimentación. Podemos citar el trabajo de Lucía Bascuñán (1991) que analiza la alimentación cotidiana y de fiesta en un pueblo del estado de México. El objetivo de Bascunán es ver el impacto nutricional de la alimentación festiva a partir de la descripción de la alimentación como parte del conjunto de rasgos socioculturales y económicos. Otro trabajo que vale la pena mencionar es el de Ma. de la Luz Valle (1998) quien hace un análisis de la alimentación de Xochimilco con el fin de mostrar su importancia como elemento ritual.

En lo que va de siglo encontramos en México un auge hacia los estudios de la alimentación usando metodología antropológica. Ya sea antropólogos se han interesado por la comida, o bien profesionales de la salud y la nutrición que han buscado en la antropología una nueva forma de estudiar el comportamiento alimentario; en éstos últimos, el punto central siempre tiene que ver con el mejoramiento de las formas de comer para la prevención de enfermedades, o bien para superar los rezagos nutricionales, y proponer soluciones más acordes a los hábitos de la población objetivo. Algunos de estos trabajos, fueron presentados en un seminario sobre antropología y nutrición en el 2004, y publicados recientemente (BERTRAN; ARROYO, 2006).

El uso de la antropología para el análisis de los hábitos alimentarios se ha utilizado a menudo con el fin de explicar la situación alimentaria de un grupo, y obtener así elementos que ayudaran mejorarla. Esto fue así en el inicio del interés de la antropología por la alimentación, y es así en al actualidad. En este sentido, la antropología de la alimentación puede considerarse como una aportación a la antropología aplicada y ha tenido dos grandes preocupaciones: primero sobre los problemas de subsistencia, es decir de ver si toda la población comía de manera 
suficiente; posteriormente como un asunto relacionado con la salud a partir del auge de la educación y promoción del autocuidado la salud.

En la actualidad, los procesos macrosociales como la globalización y sus manifestaciones en la disponibilidad y acceso a los alimentos, la difusión de conocimientos, la migración, o bien el aumento en la monetarización de la economía en todos los sectores sociales de manera acelerada en países con economías emergentes como México, hacen que sea imperativo un análisis de cómo todo esto afecta la vida cotidiana de la gente y sus actividades para la subsistencia.

\section{México y la Ciudad de México}

Según el último Conteo de Población de 2005, en México viven un poco más de 100 millones de habitantes de los cuales hay una proporción ligeramente mayor de mujeres que de hombres, debido en buena medida a la migración hacia Estados Unidos.

Aunque la esperanza de vida ha aumentado drásticamente desde los años 70, pasando de 61 a 75 años en el año 2000, la población mexicana sigue siendo joven; el $48 \%$ de la población es menor de 15 años. La tasa de fecundidad ha pasado de 7.3 hijos por mujer en la década de los sesenta (el máximo histórico) a 2.1 en promedio en la actualidad. Sin embargo, las diferencias por estrato socioeconómico son notables al grado que en las mujeres con niveles de escolaridad más bajos, tienen una fecundidad del doble del promedio. ${ }^{1}$

\section{CUADRO 1 - Demografía México}

\begin{tabular}{|l|l|}
\hline Población (2005) & 103.263 .388 \\
\hline Hombres & 50.249 .955 \\
\hline Mujeres & 53.013 .433 \\
\hline Población estimada (2008) & 106.682 .518 \\
\hline Crecimiento anual (2000-2005) & $1,0 \%$ \\
\hline Fecundidad (2008) & 2,1 \\
\hline Esperanza de vida (2008) & 75.1 años \\
\hline Población menor de 15 años (2005) & $48 \%$ \\
\hline Area Metropolitana Ciudad de México (2005) & 19.231 .829 \\
\hline Población de habla indígena (2005) & $30 \%$ \\
\hline Lenguas indígenas & 62 \\
\hline
\end{tabular}

Conteo Población (INEGI, 2005). 
En los últimos 50 años, México ha reducido de manera drástica su población rural. En el año 2000 sólo el $25 \%$ de la población vivía en localidades menores a 2500 habitantes, mientras que en 1960 era casi el 50\%. Las actividades primarias de la economía ocupan sólo al 16\% de la Población Económicamente Activa (PEA). El crecimiento de la urbanización se ha hecho básicamente a expensas de la migración rural-urbana a los grandes núcleos urbanos, y en menor medida a las ciudades medias mayores de 100 mil habitantes (INEGI, 2000).

Otro elemento interesante ha sido la paulatina incorporación de las mujeres al mercado de trabajo. En 1960 el 18.1\% de la PEA eran mujeres, mientras que en el año 2000 sobrepasa el 30\%. Este dato hay que tomarlo con reserva ya que las mujeres participan en una gran cantidad de actividades económicas en el sector informal como empleadas domésticas, comercio a pequeña escala y venta de comida preparada entre otras. Estas características demográficas del país se dieron primero en la ciudad de México, centro político y económico del país aún desde la época prehispánica.

Para hacer un texto sobre la ciudad de México, es casi inevitable empezar sin señalar su gran tamaño; es la segunda concentración urbana del mundo después de Tokio. Su zona metropolitana está integrada por el Distrito Federal con 16 delegaciones políticas, 58 municipios del Estado de México y uno del Estado de Hidalgo. En conjunto, viven en la megaurbe 25 millones de personas, de las cuales sólo alrededor de 8 millones corresponden a la zona administrativa del Distrito Federal. El Distrito Federal y los municipios tienen administraciones políticas diferentes. Los datos que vamos a usar para contextualizar este trabajo serán exclusivamente del Distrito Federal.

El crecimiento demográfico de la Ciudad de México se debe en buena medida a la migración, siempre constante, aunque ha tenido periodos más acelerados. A finales del siglo XIX, la migración tuvo un gran impulso cuando el deterioro de las condiciones económicas de las zonas rurales coincidió con el auge de económico de la ciudad de México (ACEVEDO, 1995). Esta migración mantuvo un ritmo sostenido aunque moderado hasta los años treinta del siglo XX. Una década después la migración hacia la ciudad de México aumentó notablemente como resultado del proceso de industrialización en el país que requirió mano de obra para llevarse a cabo. Las políticas de apoyo a la industria, incluyeron el abandono de programas para el campo, lo que aceleró la búsqueda de mejores opciones para la población 
rural, que encontró en la ciudad de México, no sólo una opción de empleo, sino también de bienes y servicios. El crecimiento demográfico desbordado de la ciudad de México, es pues resultado de la industrialización como en muchas otras partes del mundo, pero también del poco apoyo a las actividades económicas en las zonas agrícolas. Es también resultado de que el proceso de urbanización se haya hecho básicamente a expensas de la ciudad de México, y no de una política de desarrollo regional. La tasa de urbanización en México ha pasado de 54.9\% en 1965 a 74.4\% en el año 2000. Se estima que esta tasa aumente a $81.9 \%$ en 2030. (BERTRAN; PÉREZ-LIZAUR; ROSELLÓ, 2006).

\section{Diversidad social, económica y cultural}

La migración a la ciudad de México, así como las diferencias sociales y económicas, hacen que la población sea muy diversa. La clasificación por estratos es complicada, en tanto es resultado no sólo del ingreso, la escolaridad o el empleo, sino de la combinación de factores económicos, sociales y étnicos. Sobre este último aspecto, por ejemplo, hay que señalar que México es un país con el 10\% de su población clasificada como indígena, ${ }^{2}$ y es la que siempre se ubica en los estratos más bajos de la sociedad en la que vive, ya sea en una zona rural o en las ciudades; su propia condición étnica los ha dejado fuera del desarrollo nacional al grado que para mejorar lo primero que deben buscar es dejar de ser indígenas. Esta diversidad de formas de organización, sin duda responde a diferencias económicas, pero también a diferencias culturales, lo que en términos alimentarios es importante en tanto se expresa en diferencias en acceso a servicios de abasto, en formas de preparación, en ideas sobre los alimentos o en lógicas de gasto y consumo de alimentos.

En términos socioeconómicos, la diversidad se expresa en la escolaridad o el empleo, o una combinación de ambos. La población del Distrito Federal tiene en promedio 10.8 años de escolaridad, dos más que la media nacional. Los mejores empleos en las zonas urbanas, en su mayoría los ocupan quienes tienen los niveles de escolaridad más altos, y son quienes a su vez tienen los niveles de ingreso más altos (INEGI, 2005).

\section{Alimentación y nutrición en México}

En México, las encuestas nacionales de nutrición entre 1988 y 2006 muestran un rápido aumento de la obesidad en la población a un nivel tal que hoy, cerca 
del $70 \%$ de la población adulta tiene algún grado de sobrepeso. Las primeras causas de enfermedad y muerte, seguida por las enfermedades cardiovasculares y algunos tipos de cáncer (INSP, 2006). Estas enfermedades, si bien son provocadas por múltiples factores, tienen como uno de los principales factores de riesgo la alimentación; el rápido crecimiento de la obesidad y las evidencias médicas dejan clara su relación con el perfil epidemiológico. Al mismo tiempo, la encuesta también reveló que si bien se han hecho avances en la disminución de la desnutrición crónica todavía hay $12.7 \%$ de los niños menores de 5 años que tienen baja talla para su edad. Es decir, todavía no ha superado sus problemas de desnutrición cuando enfrenta, a menudo incluso en la misma población, serios problemas de salud pública relacionados con la mala alimentación. Los datos sobre consumo de alimentos obtenidos en la Encuesta Nacional de Nutrición 1999 (INSP, 2000), señalaron que la población mexicana tiene un consumo excesivo de alimentos de alta densidad energética, ${ }^{3}$ mayor consumo de grasas que la recomendación, la mayoría de los aceites que se consumen son ácidos grasos trans, ${ }^{4}$ alto consumo de azúcares concentrados, y un bajo consumo de frutas y verduras: $120 \mathrm{~g}$ vs. $400 \mathrm{~g}$ que es la recomendación. De hecho, desde los años sesenta se observó un aumento en el consumo aparente de de grasas y azúcares.

\section{Gráfico 1 - Consumo aparente de azúcar (per cápita/dia) México 1960-97}

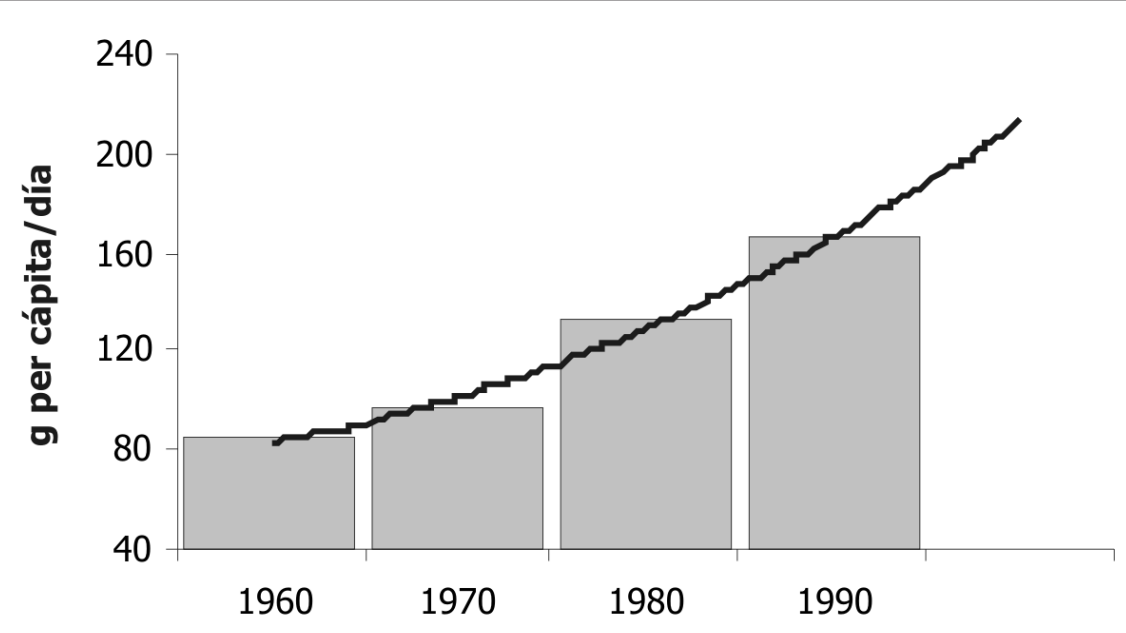

A partir de las siguientes fuentes: Revista de Comercio Exterior 1971:67; INEGI: El sector alimentario en México. Cuarto Informe de Gobierno, 1993 y FAO 1998 


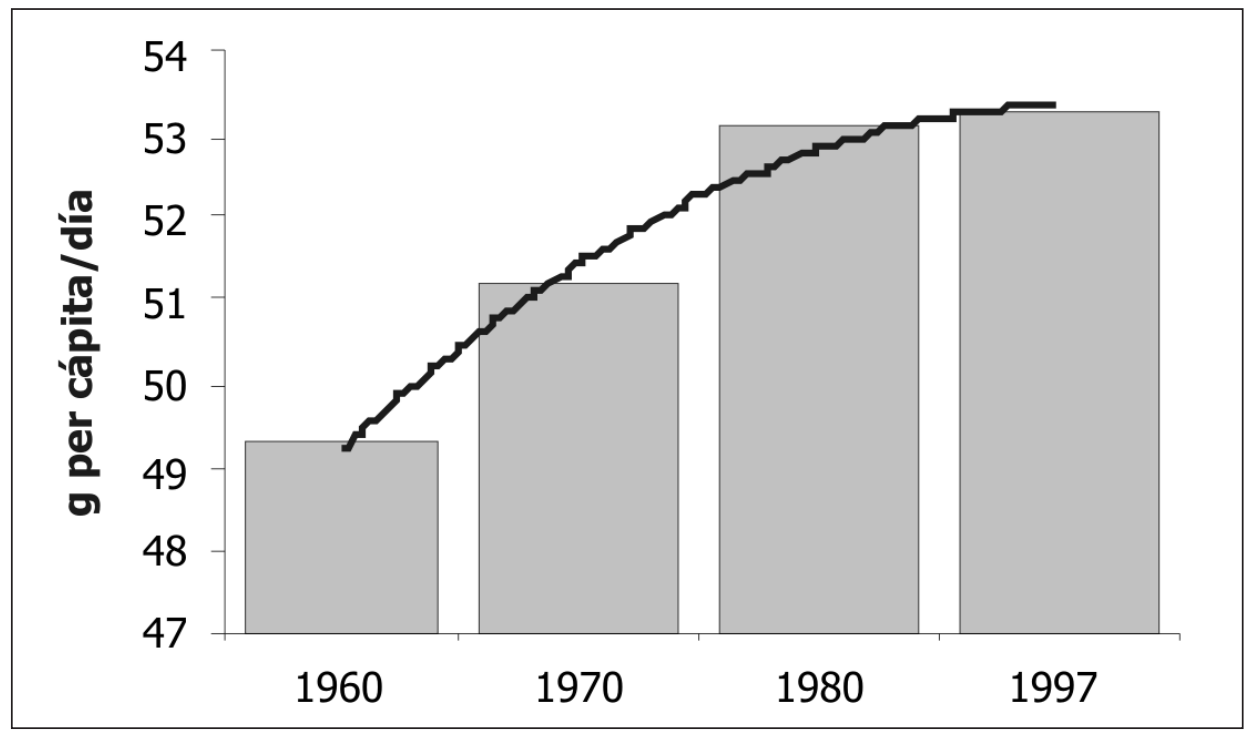

Por su parte, los datos sobre la ciudad de México reportados en la Encuesta Urbana de Alimentación 2002 (INNSZ, 2003) encontraran que en la capital el consumo de lípidos es en promedio de $33 \%$ del total de energía de la dieta, cuando la recomendación es alrededor del $25 \%$ para población normal adulta. ${ }^{5}$ Esta misma encuesta mostró que el porcentaje de sobrepeso y obesidad en la población adulta entre 18 y 49 años es del 60\% y de la población escolar de 34.5\%.

Por lo que respecta a la alimentación, dada la diversidad en México es difícil hacer una descripción general. Las formas de comer dependen en buena medida de los recursos, económicos o del campo según el caso, y de las posibilidades de acceso. Las diferencias se expresan también términos culturales: en la parte norte se come más carne y productos de trigo mientras que en el sur la presencia de la dieta tradicional indígena es más común. En la costa los guisos de pescado son más comunes que en el interior donde su consumo es esporádico en la población general. Sin embargo, aún con estas diferencias, podemos delinear una serie de rasgos en común.

El alimento de mayor consumo en México es el maíz, que se consume generalmente en forma de tortillas de masa, aunque también se hacen otras preparaciones de masa pero que no suelen ser de consumo cotidiano. Diariamente 
se hacen entre dos y tres comidas estructuradas al día: el desayuno y la comida, y a veces la cena. La estructura de la comida diaria del mediodía consiste en sopa, arroz o pasta, carne con salsa picante (chile y tomate), tortillas, frijoles, agua de sabor o refresco. La carne más común para los guisados de la comida es la de pollo, pero también se hacen guisados de carne de puerco. En lugar de carne es común que se use queso, huevo o algunas verduras.

Para el desayuno huevo, cereal, leche, pan, queso, fruta. A menudo, también los sobrantes del día anterior se comen en la mañana. Por la noche fruta, pan, leche o café es lo cotidiano. Hay casos donde se hace un desayuno muy ligero, a veces sólo café con pan o tortillas, un almuerzo a media jornada de trabajo que tiene los mismos alimentos que el desayuno fuerte, y una comida al terminar el horario laboral a media tarde. Los horarios dependen en gran medida de las actividades laborales, la distancia entre la casa y el trabajo, el tipo de trabajo y la organización doméstica. Por ejemplo, cuando hay hijos pequeños es más común que haya una estructura horaria, mientras que cuando los hijos ya son grandes y trabajan la hora de la comida puede ser inexistente y cada quién come según sus necesidades.

En la medida que la alimentación es más variada la proporción del maíz en la dieta ha disminuido aunque sigue siendo el alimento de mayor consumo. En las zonas rurales pobres, todavía hoy, hay sectores de población que tienen una dieta muy monótona basada en maíz, frijol, salsa picante, algunas verduras y ocasionalmente carne u otros productos de origen animal. En estos casos, el maíz en forma de tortillas es el alimento base, mientras que en la medida que la dieta se diversifica las tortillas pasan a ser acompañantes.

Por lo que se refiere a los productos de origen animal, hay que destacar la elevación en la ingestión de carne de pollo en las últimas décadas, y la disminución de la importancia del cerdo en la dieta. La carne de res se come menos, y prácticamente en las clases más acomodadas. Sin embargo, es importante señalar que en este sector se come con reserva ya que se considera que no es saludable. El huevo, la leche y el queso es de consumo cotidiano; a los niños, en la medida que haya recursos para hacerlo, se les permite y fomenta que tomen leche, incluso hasta un litro al día. El consumo de carne en las fiestas contrasta con el de la comida cotidiana. Las celebraciones tienen como platillo central la carne ya sea de cerdo, de pavo (para comer con mole) o la barbacoa de carnero. 
En la medida que la agricultura es una actividad cada vez menos frecuente, la compra de alimentos es la principal forma de obtenerlos. La autoproducción, que en otras épocas había servido para la alimentación básica ha disminuido y permanece tan solo en algunas zonas rurales marginadas. En algunos casos, producir el propio maíz para comer es más caro que comprarlo.

Desde los años sesenta en México la disponibilidad de alimentos ha ido en aumento. Según los datos de FAO (2003), el suministro de energía per cápita pasó de 2626 kilocalorías por habitante en 1965 a $3159 \mathrm{kcal} / \mathrm{hb}$ en el año $2000,{ }^{6}$ de hecho desde los años ochenta la cantidad de energía disponible por persona en el país no ha bajado de $3000 \mathrm{kcal}$. Como resultado se observa un aumento paulatino en el consumo de aceite, azúcar, carne y lácteos. ${ }^{7}$ Evidentemente, en zonas como la ciudad de México, que maneja buena parte del abasto no sólo de la megaurbe, sino también de varios estados de la República, la cantidad de alimentos disponibles es mucho mayor. Históricamente, el abasto en la ciudad de México ha sido bien calificado, en tanto no se han presentado situaciones de desabasto de alimentos. Más bien al contrario; del total de alimentos que recibe el 15\% se redistribuye hacia otros estados. El abasto en la ciudad se ha configurado bajo el esquema más clásico de una central de abasto, que a su vez distribuye a millares de puntos de venta a los que acuden los consumidores. Estos puntos de venta son de diversa índole: tiendas de abarrotes, puestos en la vía pública, mercados sobre ruedas y fijos, tiendas especializadas en pollo, carne, verduras, lácteos, y más recientemente los supermercados (TORRES, 2000). Un punto importante que señala Torres (2000) sobre los supermercados es que su expansión hacia zonas más populares de la ciudad ha generado una especie de homogeneización en el consumo de alimentos, y una idea de acceso más amplio a otros sectores de la sociedad que tradicionalmente compraba en las pequeñas tiendas de sus barrios. La homogeneidad en el consumo, sin embargo, no es en realidad tal, pues la calidad de los alimentos es distinta según los precios. Así, por ejemplo, las poblaciones de más bajos recursos tienen acceso a comer carne y embutidos como los sectores altos, sólo que con un porcentaje de grasa mayor. Esto, entre otros factores explica también que la obesidad en México, como en otros países en vías de desarrollo, ha ido en aumento de manera más rápida en los sectores de más bajos recursos. El sobrepeso y las enfermedades asociadas ya no son una condición de las clases altas; en todo el mundo, y en particular en los países en vías de desarrollo, una vez que se ha superado la escasez, 
aparece la obesidad entre la población más pobre. (PEÑA; BACALLAO, 2000). En el caso concreto de México un estudio sobre el precio de los alimentos desde los años setenta, muestra que los alimentos industrializados, especialmente los de alta densidad energética han disminuido de precio de manera gradual desde los 70, al mismo tiempo que las frutas y verduras se han encarecido (ORTIZ, 2006). La población que tiene acceso a estos alimentos de alta densidad energética como los refrescos, pastelitos, panes, galletas, frituras, alimentos preparados, son un sector de la población donde este acceso es reciente. Han pasado en poco tiempo de una dieta monótona y escasa, a una dieta más variada con acceso a alimentos diversos, muchos de ellos industrializados. En este sentido, algunos autores han señalado que el consumo de alimentos en población que ha pasado escasez tiende hacia la sobrealimentación. (GONZÁLEZ-TURMO, 2002). Para esta población, adicionalmente, los alimentos industrializados y urbanos tienen el significado de alcanzar la modernidad. Representan una forma de mejorar socialmente, una forma de movilidad social en una sociedad con alta estratificación. Esta característica, como veremos a continuación, tiene determinantes sociales y culturales presentes históricamente en México.

\section{Alimentación e identidad}

La estratificación social en México es y ha sido un elemento característico de la sociedad mexicana y tiene efectos en la alimentación. A la llegada de los españoles la población estaba dividida en rangos, tanto dentro de los propios grupos indígenas como en las relaciones entre ellos. Había grupos dominantes que exigían el pago de tributo, en muchos casos con alimentos. Los mexicas mostraban su superioridad con respecto a otros grupos también con las formas de alimentarse: consideraban que comían mejores tortillas y veían a los otomís como pueblos atrasados porque no sabían cosechar el maíz a tiempo o a los purépechas como bárbaros pues comían tortillas duras (PILCHER, 2001). Al interior de un grupo, la comida de los nobles y sacerdotes era diferente del resto de la población mostrando así su condición superior. También en las fiestas, la comida adquiría significados de estatus pues el anfitrión la hacía lo más suntuosa posible. (GONZÁLEZ DE LA VARA, 1996).

La estratificación social en México se agudizó durante la época colonial, tanto por las condiciones en el sistema socioeconómico establecido por los españoles 
como por la diversidad de la población: indígenas, españoles y negros, éstos últimos traídos para aumentar la mano de obra. Esto generó una gran cantidad de grupos resultado de las mezclas entre ellos. En la parte superior de este sistema se ubicaban los españoles y de ahí iban descendiendo en la escala social según las mezclas y la presencia de sangre indígena o negra. Según Jeffrey Pilcher, historiador norteamericano que analiza el papel de la comida en la construcción de la identidad nacional mexicana, la alimentación tiene un papel muy importante como marcador social en la sociedad novohispana, para mostrar la pertenencia a una clase o la aspiración (PILCHER, 2001). Es decir, las formas de comer se usan tanto como elemento para mostrar la pertenencia a un estrato como para aparentar la pertenencia a otro y por tanto dejar el de origen. Así, la alimentación como rasgo social puede ser tomada como un elemento integrador a otro grupo, no sólo un diferenciador. Los indígenas usaban la alimentación como rasgo integrador cuando querían aparentar no serlo y pertenecer a otro estrato. Para pasar de indígena a mestizo urbano era suficiente con vivir en la ciudad y empezar a comer pan de trigo y vino. Pilcher sostiene que la importancia de la alimentación en la colonia como rasgo de identidad es resultado de la estratificación social así como de la imposibilidad de identificar racialmente a los grupos, con lo que ciertos rasgos de vuelven importantes (PILCHER, 2001).

Después del movimiento de Independencia, cuando surge la República Mexicana como tal, la población indígena pasa a tener el mismo estatus jurídico que cualquier otro mexicano, eliminando así las diferencias establecidas en el régimen colonial. Esto no significó, por supuesto, ninguna condición real de igualdad ni mucho menos, así como tampoco significó ningún respeto a sus características étnicas. Al contrario desde el inicio se buscó la integración de la población indígena, básicamente por dos motivos: por un lado, México debía construir una identidad nacional homogénea correspondiente a su nacimiento como nación; por otro, desde el principio se planteó la preocupación por mejorar las condiciones de la población indígena. En este contexto, algunos autores señalan que en el siglo XIX las políticas hacia los indios buscaron su desaparición. Incluso Benito Juárez, él mismo indio zapoteco, consideraba que la cultura indígena era el motivo del retraso de la población e impulsó la integración (DEL VAL, 1993).

Estas políticas sobre los indígenas fueron representadas en muchas ocasiones sobre asuntos relacionados con su alimentación. Durante el siglo XIX, la 
alimentación fue un tema importante en las políticas nacionales hacia la población indígena. Por una parte, había un reconocimiento de los problemas nutricionales de la población y por tanto todos los gobiernos tomaron una postura al respecto. El común denominador coincide plenamente con las políticas hacia la población indígena, es decir para que tengan desarrollo deben dejar de ser indígenas y por tanto dejar de comportarse como tales, incluyendo la alimentación. Por ejemplo, durante el periodo de Benito Juárez como presidente, se planteó hacer programas de educación para las mujeres, con el objetivo de "civilizar a las masas, sobretodo a los indios" (PILCHER, 2001, p. 103). La idea es que las mujeres promovieran otro comportamiento desde el ámbito doméstico. Sin embargo, una cosa era lo que decían los políticos y otra lo que pasaba en la sociedad, ya que era común que provocara conflictos sociales. La población no iba a dejar de ser indígena sólo por decreto gubernamental; más bien iba a dejar de serlo como una manera de mejorar socialmente mientras la sociedad lo reconociera así. Es justo en esta época, cuando aparecen toda una serie de modas extranjeras en México, donde la cocina es buena muestra. Hay un gusto especial por la cocina europea, y en los banquetes y comidas de las clases acomodadas se ofrecen platillos franceses, ingleses y de otros países de Europa (RABELL JARA, 1996). Según Pilcher varios documentos de la época muestran el desprecio de la sociedad decimonónica por el chile y la comida indígena. Al mismo tiempo, la aparición de México como nación, generó la necesidad de crear una serie de rasgos nacionales de identidad, y en algún momento la cocina se volvió uno de ellos. Así se publicaron algunos recetarios como el Cocinero Mexicano, que apareció por primera vez en 1831 con lo que se usó a la cocina como un elemento de identidad nacional. Sin embargo, los platillos de maíz nunca pudieron quitarse el estigma de identificar a las clases bajas (PILCHER, 2001). De esta manera el siglo XIX transcurre con unas ideas contradictorias sobre la comida tradicional mexicana que flotan en la sociedad: por un lado se usa para buscar las raíces nacionales y construir la identidad, pero por otro está asociada a la pobreza y los indígenas. Además, las políticas sobre la población indígena son muy claras: hay que civilizarlos e integrarlos a la sociedad nacional.

Las ventajas de la dieta indígena mexicana no fueron reconocidas sino hasta los años cuarenta, cuando aparece el Instituto Nacional de la Nutrición ${ }^{8}$ y se empiezan a estudiar sus características bromatológicas. La creación del INN en 1944 surge por la necesidad de mejorar la situación nutricia de la población dadas 
las condiciones de desnutrición en que se encuentran. Este fue uno de los puntos centrales en los trabajos del Primer Congreso Indigenista Interamericano donde los diferentes especialistas reunidos señalan que la alimentación de los indígenas es inadecuada para su desarrollo biológico y en la mayoría de los grupos se advierte la presencia de desnutrición; pero también expresan que es necesario conocer científicamente las características de la dieta. Así, se empezaron las investigaciones sobre la distribución de la desnutrición en el país, las técnicas de preparación de alimentos, los efectos de la dieta tradicional en el organismo que fueron mostrando que la alimentación indígena en sus características básicas proporciona suficientes elementos para desarrollarse, siempre y cuando se coma en cantidad suficiente (AGUIRRE BELTRÁN, 1986). En términos nutricios, la dieta tradicional indígena es una buena fuente de energía e hidratos de carbono, de proteína en la combinación del maíz y frijol, de vitaminas y minerales si hay suficiente consumo de frutas y verduras, así como en el consumo cotidiano de chile; tiene además ventajas como ser buena fuente de calcio por la nixtamalización del maíz, baja en grasa en su forma original y tener un buen contenido de fibra.

Sin embargo, la población en la medida que tiene acceso a los recursos económicos, prefiere alimentos industrializados y "modernos" que les permitan ir mostrando su mejoramiento social. Esto, a pesar de las reivindicaciones indígenas y de las ventajas dietéticas de la alimentación original, parece ser resultado de una idea sistemáticamente presente en la sociedad, y expresada abiertamente hasta hace muy poco tiempo en las políticas públicas: para mejorar y desarrollarse es preferible dejar de ser indígena. En este sentido, la alimentación ha tenido un papel importante a lo largo de la historia, tanto por sus significados sobre la estratificación social como por la creencia, hasta hace apenas 50 años cambiada, que la dieta indígena no era adecuada. Así, la alimentación indígena parece tener un papel como rasgo de identidad pero en sentido contrario como una forma de dejar de ser indígena y buscar integrarse a la sociedad mayor (BERTRAN, 2005). Esta característica, junto con la disminución de precio de los alimentos de alta densidad energética puede explicar la alta presenciad de sobrepeso y obesidad en México. Sin embargo, al mismo tiempo que crece la obesidad han aparecido también las ideas sobre las ventajas de la delgadez, tanto en términos de salud como de imagen. Es posible, que esto sólo esté presente en un sector de la población, quizá porque tiene mejores niveles educativos, o quizá como una manera de distinguirse de los estratos bajos. 


\section{Alimentación, salud e imagen corporal}

El impacto de la comida en la imagen, la salud y el bienestar es una de los elementos presentes en la actualidad en las elecciones alimentarias. Si bien es cierto, que las condiciones socioeconómicas que determinan las posibilidades de acceso y de tiempo de preparación de los alimentos, son fuertes limitantes de las maneras de comer, no por ello deja de ser importante, al menos como ideal que la comida sea saludable. Los medios de comunicación, la publicidad, los mensajes del sistema médico hacen eco de esta preocupación social por la relación entre alimentos, salud, bienestar e imagen corporal.

El desarrollo de las ciencias de la nutrición, la industria y la difusión de la información entre comida y salud ha tenido impacto en las percepciones y prácticas alimentarias (FISCHLER, 1995). Esto se expresa en las formas de comer y en los atributos y significados que se otorgan a los alimentos: que engordan, adelgazan, son buenos para los niños, malos para las mujeres embarazadas, etc. Los significados atribuidos a los alimentos, y en si mismo todo el proceso alimentario son rasgos que se expresan de manera diferente según el estrato social, y de hecho puede ser usado como una forma de identidad en las sociedades complejas con alta estratificación. Sobre este asunto, Jack Goody (1996) señala que donde hay más estratos sociales, los sistemas culinarios serán más elaborados, pues se constituyen como una forma de identificar a cada estrato, o mejor dicho, de diferenciarse entre sí. Un punto central en la estratificación son los rasgos que la caracterizan y por tanto, los individuos buscan tener unos u otros para mostrar la pertenencia a un grupo; también sirven para señalar que se ha dejado de pertenecer para entrar en otro de mayor grado en la escala social, como parece ser el caso de la población indígena que a través de la comida busca mostrar que ha entrado a la sociedad mayor (BERTRAN, 2005). Es en este sentido que Mintz (1996) dice que los alimentos son un marcador social.

La relación entre alimentación, salud y corporalidad o imagen, igualmente se expresan de diferente manera en los estratos sociales. Sobre el asunto de la alimentación y la imagen corporal Bourdieu señala que la distinción de clase está no sólo en lo qué se come, y cómo, sino también en los efectos corporales que provocan, en la imagen que proyectan. En este sentido, el cuerpo expresa una imagen ideal que se expresa en términos estéticos. La revisión de la literatura al respecto realizada por Reischer y Koo (2004), muestra que el cuerpo se ha 
estudiado no sólo como símbolo en el sentido que es un vía de expresión, sino también un medio de relación social, de interacción social. Las autoras señalan que las últimas propuestas teóricas al respecto, consideran al cuerpo como un aspecto fundamental del actuar del yo (self), tratando así de superar la dicotomía del asunto del yo como subjetivo versus el cuerpo como objetivo, para dirigir la atención, a menudo ignorada, del papel del cuerpo en la acción social.

En el caso de México, a pesar de las diferencias sociales, esta preocupación está presente en todos los sectores urbanos, aunque no se manifiesta del mismo modo. En los sectores más bajos la preocupación por la imagen es importante, como una cuestión de salud y también como un asunto de vanidad. En contraste, en los sectores medios y altos, la imagen y la salud son claramente percibidos como símbolos de bienestar, como un ideal social. Un cuerpo bello es sano, refleja bienestar, no sólo por cómo se siente sino, y básicamente, por cómo se ve. En este sentido, como en todo el mundo, la delgadez es el ideal a conseguir lo que se obtiene fundamentalmente por la comida saludable, sobretodo baja en grasa, haciendo ejercicio, tomando mucha agua, sometiéndose a tratamientos corporales incluyendo la cirugía (BERTRAN, 2007). La corporalidad es un agente social que señala las posibilidades económicas, culturales y de tiempo.

Sin embargo, la población mexicana se enfrenta a una de las paradojas alimentarias más relevantes del mundo contemporáneo, de manera quizá más aguda que en otros lugares. Contreras (2002) señala que en las sociedades actuales al mismo tiempo que la delgadez es el ideal corporal social el sobrepeso y la obesidad aumentan; "la gente se sabe gorda y se sueña delgada" (CONTRERAS, 2002, p. 282) ¿Cómo explicar esto en México cuando más del 70\% de la población adulta tiene algún grado de sobrepeso? Por una parte porque al mismo tiempo que se busca la delgadez y la salud, el bienestar está también se promueve el placer y el gusto, el consumo de alimentos industriales que además de ser baratos dan prestigio y cumplen los ideales de modernidad. Por lo que corresponde al hedonismo, como nunca antes, la cocina, la gastronomía, la búsqueda de alimentos nuevos, de sabores nuevos está presente como parte de la vida diaria, en todos los niveles. En los sectores altos se expresa en las nuevas propuestas gastronómicas de la industria restaurantera que proponen más que una salida a comer una experiencia sensorial; en los sectores bajos el acceso reciente al fast food americano (hamburguesas, pizzas, sushi) permiten acceder a una nueva 
y moderna forma de vida. Así en la alimentación contemporánea en México conviven en placer y el hedonismo, con el control del consumo para guardar la línea y la salud. La industria alimentaria en cualquier caso tiene opciones para todo, incluso para la experiencia del gusto sin los efectos en el cuerpo como los alimentos light, bajos en grasa, sin azúcar, etc. Pero en la promoción de todos estos alimentos, se emiten multitud de mensajes que son contradictorios: el placer, el control del placer, la salud, el gusto, la imagen, etc.

Sin embargo, el cuidado excesivo del cuerpo y la delgadez tampoco son vistos como algo adecuado. En la ciudad de México, por ejemplo, las mujeres se preocupan por la apariencia de sus hijas, de que no estén gordas, al mismo tiempo que procuran que gocen con la comida y no se obsesionen con el peso y caigan en un trastorno alimentario (BERTRAN, 2007). Esto quizá es resultado de la postura médica que al mismo tiempo llama la atención sobre los peligros de la obesidad y advierte del riesgo por los trastornos alimentarios como la anorexia y la bulimia. Igualmente, esta contradicción está presente en los medios de comunicación que promueve unos cánones de belleza donde la delgadez es la norma, pero denuncia los casos de mujeres con trastornos alimentarios por alcanzar el peso que se requiere para ser modelo. Recientemente, han aparecido en las noticias discusiones sobre el tema a raíz de la muerte de varias modelos por anorexia.

Por otro lado, en los sectores más altos la población está preocupada por las formas de producir y transformar los alimentos. En este sentido, asistimos también al surgimiento de movimientos que buscan "lo natural" y "lo orgánico" como una manera de huir de la industria. Según Mintz (1996) en Estados Unidos el interés por lo "natural" ha tenido sus altibajos, pero el último movimiento hacia este tipo de alimentos parece haber coincidido con el rechazo a la guerra de Vietnam, y en general al gobierno y al sistema político y social dominante en los años sesenta. Este movimiento igualmente tuvo su impacto en México y en los años setenta aparecieron grupos de vegetarianos y otros tipos de dietas restrictivas como una manifestación en contra de los efectos de la industria en el medio ambiente y en el cuerpo. Actualmente, la búsqueda de los alimentos naturales es una manera, también, de evitar la comida industrializada, por considerarla llena de químicos que la gente percibe como elementos potencialmente dañinos. También han aparecido los productos "orgánicos" que teóricamente no están manipulados genéticamente, o no se le han agregado fertilizantes, pesticidas u 
hormonas en su proceso de producción. Estos productos, que son mucho más caros que los de producción masiva, obviamente son principalmente consumidos por los estratos medios y altos, quienes se justifican en términos de salud. Sin embargo, considerando la estratificación social en México, sería lógico suponer que marca también una distinción de clase, para diferenciarse de los estratos bajos quienes comen mayormente los alimentos industriales.

\section{Conclusiones}

La situación alimentaria actual en México está determinada por los cambios sociales, culturales y económicos de los últimos 50 años. La globalización ha tenido sus efectos en términos de la cantidad de alimentos disponibles y de la difusión de información sobre ellos. La estratificación de la sociedad mexicana ha provocado que estos cambios no sean iguales en todos los sectores sociales lo que se expresa en los significados que se le atribuyen a los alimentos y a las elecciones mismas para la comida cotidiana.

Los datos sobre la alimentación en México y los fenómenos sociales relacionados dan cuenta de la complejidad del fenómeno alimentario y de cómo los procesos macrosociales afectan las decisiones cotidianas de la gente. El análisis antropológico de la alimentación en la población mexicana ha permitido mostrar la relación entre estos procesos históricamente y en fechas recientes. Son la muestra de la utilidad de la metodología antropológica para estudiar la alimentación contemporánea, llena de contradicciones, que tienen que ver con el desarrollo del capitalismo y la sociedad de consumo, la promoción al consumo, y el acceso inmediato a él, la medicalización de la vida cotidiana, las ideas sobre el control corporal, y la imagen como un elemento de estatus.

La población se enfrenta a tener de decidir su comida considerando que quiere cuidar su salud, verse bien, comer bien, que le guste, sin generar la obsesión por la comida y la imagen; siempre claro dentro del margen de posibilidades de su estrato socioeconómico. ¿Cómo, en medio de esta complejidad, podemos ofrecer alternativas reales para mejorar la alimentación, prevenir enfermedades y mejorar la calidad de vida? En medio de las cifras epidemiológicas, éste es el gran reto para los estudiosos del tema y los planificadores de campañas de salud, lo que requiere del concurso de todas las disciplinas relacionadas con la alimentación y nutrición. 


\section{Referencias}

ACEVEDO, M.L. Los Mixtecos. In: . Etnografía contemporánea de los pueblos indígenas de México. Región Pacifico Sur. México: Instituto Nacional Indigenista, 1995. p. 81-183. AGUIRRE-BELTRAN, G. Antropología Médica. México: CIESAS, 1986.

BASCUÑAN, L. Estudio de la alimentación habitual y de la comida de las fiestas de la población de San Miguel Tlaixpan, Texcoco. Una propuesta metodológica. México: Tesis Universidad Iberoamericana, 1991.

BEALS, R.; HATCHER, E. The Diet of a Tarascan Village. América Indígena, v. III, n. 4, s/p, 1943.

BERTRAN, M. Cambio alimentario e identidad de los indígenas mexicanos. México. Programa Universitario México Nación Multicultural. Publicaciones y fomento editorial. UNAM, 2005.

BERTRAN, M. Significados socioculturales de los alimentos en la Ciudad de México. In: BERTRAN, M.; ARROYO, P. (Ed.). Antropología y Nutrición. México: Fondo Nestlé para la Nutrición. Funsalud. Universidad Autónoma Metropolitana-Xochimilco, 2006.

BERTRAN, M. Alimentación, cultura y salud en la ciudad de México. Treball de recerca de segon any presentat en el programa de Doctorat en Antropología Social i Cultural (20052007). Facultat de Geografía i Història. Universitat de Barcelona, 2007.

BERTRAN, M.; ARROYO, P. (Ed.). Antropología y Nutrición. México: Fondo Nestlé para la Nutrición. Funsalud. Universidad Autónoma Metropolitana-Xochimilco, 2006.

BERTRAN, M.; PEREZ LIZAUR, A.B.; ROSELlÓ, M.E. Postura del Colegio Mexicano de Nutriólogos sobre los problemas prioritarios de alimentación y nutrición en el país y los programas para atenderlos. México: Colegio Mexicano de Nutriólogos, 2006.

BONFIL, G. Diagnóstico sobre el hambre en Sudzal, Yucatán: Un ensayo de antropología aplicada. México: Instituto Nacional de Antropología e Historia, 1962.

BOURDIEU, P. La Distinción. Criterio y bases sociales del gusto. Barcelona: Taurus, 2002.

CONTRERAS, J. La obesidad: una perspectiva sociocultural. Form Contin Nutr Obes, v. 5, n. 6, p. 275-86, 2002.

CONTRERAS, J.; GRACIA, M. Alimentación y cultura. Perspectivas antropológicas. Barcelona: Ariel, 2005

DAMIAN, A.; BOLTVINIK, J. Evolución y características de la pobreza en México. Comercio Exterior, v. 53, n. 6, p. 519-531, 2003.

DEL VAL, J.M. El indigenismo. In: ARIZPE, L. (coord.). Antropología Breve de México. México: Academia de la Investigación Científica. Centro Regional de Investigaciones Multidisciplinarias-UNAM, 1993. 
ESPAÑA, A. La alimentación y la nutrición. In: La antropología en México: Panorama Histórico. Tomo III. México: Instituto Nacional de Antropología e Historia, 1988. p. 81-94.

FAO. Perfiles nutricionales por países. México. 2003. Disponible en: ftp://ftp.fao.org/es/esn/ nutrition/ncp/mexmap.pdf Fecha de consulta 15 de febrero de 2007.

FISCHLER, C. El (h)omnívoro. El gusto, la cocina y el cuerp. Barcelona,:Anagrama, 1995. GAMIO, M. Hacia un México nuevo. México: Instituto Nacional Indigenista, 1987.

GONZALEZ DE LA VARA, F. La cocina mexicana a través de los siglos. Tomo II. Época prehispánica. México: Clío, Fundación Herdez A.C., 1996.

GONZÁlEZ TURMO, I. Comida de pobre, pobre comida. Somos lo que comemos. In: GRACIA, M. Estudios de alimentación y cultura en España. Barcelona: Ariel, 2002. p. 299-316.

GOODY, J. Cocina, cuisine y clase. Estudio de sociología comparada. Barcelona: Gedisa Editorial, 1995.

INEGI. XII Censo Nacional de Población y Vivienda 2000. Aguascalientes, México: Instituto Nacional de Estadística Geografía e Informática, 2001.

INEGI. II Conteo Nacional de Población 2005. Aguascalientes, México: Instituto Nacional de Estadística Geografía e Informática, 2005.

INNSZ. (2003) Encuesta Urbana de Alimentación 2002. México: Instituto Nacional de Ciencias Médicas y Nutrición Salvador Zubirán.

INSP. Encuesta Nacional de Nutrición 1999. Cuernavaca, México: Instituto Nacional de Salud Pública. Secretaría de Salud, 2000.

INSP. Encuesta Nacional de Salud y Nutrición 2006. Cuernavaca, México: Instituto Nacional de Salud Pública. Secretaría de Salud, 2006.

LOMNITZ, C.; ADLER, L. Planeación y tradición: la cultura de la alimentación en México. In: CARVAJAL, R.; VERGARA, R. La alimentación del futuro. Tomo II. México: UNAM, 1987. p. 167-184

LÓPEZ GARCÍA, J.; VERA PRÓ, R. El estudio de la alimentación iberoamericana a través de América Indígena. América Indígena, v. XLVIII, n. 3, p. 651-660, 1988.

MEAD, M.; GUTHE, C.E. Manual for the Study of Food Habits. Bulletin of the National Research Council, n. 111, 1945.

MESSER, E. Anthropological perspectives on diet. Ann Rev Anthropol., v. 13, p. 205-49, 1984.

MINTZ, S. Tasting food, tasting freedom. Boston: Beacon Press, 1996.

ORTIZ, L. et al. Evolución de los precios de los alimentos y nutrimentos en México entre 1973 y 2004. Archivos Latinoamericanos de Nutrición, n. 56, 2006. 
PEÑA, M.; BACALLAO, J. La Obesidad en la pobreza: un problema emergente en las Américas. In: PEÑA, M.; BACALLAO, J. (Ed.). La obesidad en la pobreza: un nuevo reto para la salud pública. Washington, D. C.: Organización Panamericana de la Salud, 2000. p. 3-11 (Publicación Científica, 576).

PILCHER, J.M. ; Qué vivan los tamales! La comida y la construcción de la identidad nacional. México: Ediciones de la Reina. CIESAS, CONACULTA, 2001.

RABELL JARA, R. La cocina mexicana a través de los siglos. Tomo VI. La Bella Época. México: Clío, Fundación Hérdez, A.C, 1996.

REISCHER, E.; KOO, K. The body beautiful: symbolism and agency in the social world. Annual Review of Anthropology, v. 33, p. 297-317, 2004.

SAHAGUN, FRAY BERNARDINO. Historia General de las Cosas de la Nueva España. México: Porrúa, 1985.

TORRES, F. Nuevas configuraciones del sistema de abasto alimentario de la ciudad de México. In: SÁNCHEZ ALMANZA, A. (coord.). La ciudad de México en el desarrollo económico nacional. X Seminario de Economía Urbana y Regional. México. Instituto de Investigaciones Económicas. UNAM, 2000.

VALLE BERROCAL, M.L. La alimentación en una comunidad Xochimilca: Santa Cecilia Tepetlapa. Tesis. México: Escuela Nacional de Antropología e Historia, 1998.

\section{Notas}

${ }^{1}$ Ma. Guadalupe Ledezma Basilio. Dinámica de la fecundidad en México. En http://www.eumed. net/libros/2007c/308/fecundidad.htm

${ }^{2}$ Es complicado contabilizar a la población indígena. El principal rasgo distintivo es la lengua, aunque el Consejo Nacional de Población hace estimaciones según otras características como zona de residencia, vestido, tipo de vivienda y organización social.

${ }^{3}$ La densidad energética es la cantidad de energía de un alimento por volumen. Los alimentos altos en grasa y azúcar son los de mayor densidad energética. Por ejemplo: 100 gramos de pan dulce tienen más kilocalorías que 100 gramos de pan blanco.

${ }^{4}$ Los acidos grasos trans están en grasas de origen vegetal pero tienen mayor o igual riesgo cardiovascular que las grasas animales.

${ }^{5}$ Encuesta urbana de Alimentación, 2002. Instituto Nacional de Ciencias Médicas y Nutrición Salvador Zubirán (MÉXICO, 2003).

${ }^{6}$ Datos FAOSTAT que corresponden a promedios de 3 años, i.e. 1964-66 y 1988-2000.

7 INEGI: El sector alimentario en México (CUARTO INFORME DE GOBIERNO, 1993; FAO, 1998).

${ }^{8}$ Hoy llamado Instituto Nacional de Ciencias Médicas y Nutrición Salvador Zubirán. 


\section{Anthropological approach to nutrition and bealth in Mexico}

This paper presents an overview of food in Mexico, particularly in Mexico City, from an anthropological perspective considering sociocultural and economic aspects. It begins with a brief review of anthropological studies on food in Mexico to recognize both the methodological contributions as the major problems of study. Subsequently, are presented some facts of contextualization of the country and city that frame the distinctive food and nutritional data. The following sections propose some explanations of some of the phenomena of contemporary food, where obesity is the main feature in a society of recent consumer access, while facing the cultural ideal of thinness. The data on food in Mexico and related social phenomena account for the complex nature of food and how macro-processes affect people's everyday decisions. The anthropological analysis of food in the Mexican population has been allowed to show the relationship between these processes historically and recently. They are the sign of the usefulness of anthropological methodology to study contemporary food, full of contradictions that have to do with the development of capitalism and consumer society, consumer promotion, and immediate access to it, the medicalization everyday life, ideas about body control, and image as an element of status.

> Key words: food; culture; Mexico; Anthopology. 